\title{
The Freiheitssatz for Generic Poisson Algebras ${ }^{\star}$
}

\author{
Pavel S. KOLESNIKOV ${ }^{\dagger}$, Leonid G. MAKAR-LIMANOV ${ }^{\ddagger}$ and Ivan P. SHESTAKOV $\S^{\dagger}$ \\ $\dagger$ Sobolev Institute of Mathematics, 630090, Novosibirsk, Russia \\ E-mail: pavelsk@math.nsc.ru \\ ¥ Department of Mathematics, Wayne State University, Detroit, MI 48202, USA \\ E-mail:lml@math.wayne.edu \\ § Instituto de Matematica e Estatística, Universidade de São Paulo, 05508-090 São Paulo, Brasil \\ E-mail: shestak@ime.usp.br
}

Received July 29, 2014, in final form December 22, 2014; Published online December 29, 2014

http://dx.doi.org/10.3842/SIGMA.2014.115

\begin{abstract}
We prove the Freiheitssatz for the variety of generic Poisson algebras.
Key words: Freiheitssatz; Poisson algebra; generic Poisson algebra; algebraically closed algebra; polynomial identity; differential algebra
\end{abstract}

2010 Mathematics Subject Classification: 17A30; 17B63; 17A50

Dedicated to the memory of Myung Hyo Chul (1937-2010)

\section{Introduction}

One of the classical achievements of the combinatorial group theory is the decidability of the word problem in a finitely generated group with one defining relation [10]. This result was a corollary of a fundamental statement called Freiheitssatz: every equation over a free group is solvable in some extension. For solvable and nilpotent groups, this complex of problems was studied in [16].

In the context of Lie algebras, similar statements were proved [20]. For associative algebras, the problem turns to be surprisingly difficult: over a field of characteristic zero, the Freiheitssatz was proved in [12], but the question about decidability of the word problem for an associative algebra with one defining relation remains open. One of the reasons for this is that the variety of associative algebras, contrary to those of groups or Lie algebras, is not a Schreier one, that is, a subalgebra of a free associative algebra is not necessary free. And as a matter of fact, the free algebras of Schreier varieties are usually more easy to deal with.

In [9], the Freiheitssatz was proved for right-symmetric (pre-Lie) algebras, and in [13] - for Poisson algebras over a field of zero characteristic.

There is plenty of varieties for which the Freiheitssatz is not true, e.g., so is the variety of Poisson algebras over a field of positive characteristic. One may find more examples of this kind in [14], e.g., for Leibniz algebras the Freiheitssatz does not hold (as well as for every variety of di-algebras in the sense of [8]).

Throughout the paper $\mathbb{k}$ denotes a field of characteristic zero.

A generic Poisson algebra (GP-algebra) is a linear space with two operations and one constant:

- associative and commutative product $x \cdot y=x y$;

- anti-commutative bracket $\{x, y\}$;

- multiplicative identity $1, x \cdot 1=1 \cdot x=x$,

${ }^{\star}$ This paper is a contribution to the Special Issue on Poisson Geometry in Mathematics and Physics. The full collection is available at http://www.emis.de/journals/SIGMA/Poisson2014.html 
satisfying the Leibniz identity

$$
\{x, y z\}=\{x, y\} z+\{x, z\} y .
$$

These algebras were introduced in [19] in the study of speciality and deformations of MalcevPoisson algebras.

Let $\mathrm{AC}(X)$ be the free anti-commutative algebra (AC-algebra) generated by a set $X$ with respect to operation denoted by $\{\cdot, \cdot\}$, and let $\operatorname{GP}(X)$ be the free GP-algebra with a set of generators $X$. As a linear space, $\operatorname{GP}(X)$ is isomorphic to the symmetric algebra $S(\operatorname{AC}(X))[18]$.

There are two general ways to prove the Freiheitssatz for a particular variety: one may either analyze the structure of a one-generated ideal in the free algebra (this often leads to a solution of the word problem for algebras with one defining relation) or try to construct an example of an algebra in the considered variety which is "sufficiently rich" but "relatively free". The last approach was used in [12] and [13]. In this paper, we formalize these notions in a precise form (Section 2) for an (almost) arbitrary variety. This may be helpful for study Freiheitssatz in other varieties. By "sufficiently rich" algebraic system we mean an algebra which is algebraically closed in certain sense (conditionally closed). Conditionally closed systems may not be algebraically closed in the strong sense of [1] or even existentially closed (see, e.g., [6]), but they turn to be extremely helpful for studying one-generated ideals of free algebras. Our construction of a conditionally closed generic Poisson algebra is completely different from the one in [13]. We apply general results on equations in differential algebras and then twist the standard functor relating differential algebras and Poisson algebras to obtain a generic Poisson algebra which is "relatively free". For an algebra $A$, the property to be "relatively free" means the existence of a sufficiently large lower bound for the degree of a polynomial identity holding on $A$. For example, such an algebra may have no nontrivial polynomial identities at all.

The proof that the system obtained is indeed "relatively free" is inspired by [5], but the realization of the scheme has one distinguishing feature. As in [5], we show that if a generic Poisson algebra has a polynomial identity then it has an identity of a special form. The proof is split into three main steps: (1) classification of anti-commutative polynomials that are derivations in one variable; (2) classification of anti-commutative polynomials that are derivations in all variables (Jacobian polynomials); (3) classification of Jacobian generic Poisson polynomials. The first and third steps do not essentially differ from [5], but the second step (which is almost trivial for Lie algebras) is highly nontrivial in the generic (anti-commutative) case. In particular, there are no (poly-linear) Lie polynomials that are derivations with respect to more than two variables. In contrast, one may invent an anti-commutative polynomial which is a derivation with respect to any number of variables. However, it turns out that just a limited number of anti-commutative polynomials may be Jacobian. We describe all such polynomials in Section 3.2.

\section{Conditionally closed algebras and the Freiheitssatz}

Suppose $\mathfrak{M}$ is a variety of algebras over a field $\mathbb{k}$. Denote by $\mathfrak{M}(X)$ the free algebra in $\mathfrak{M}$ generated by a set $X$. For $A, B \in \mathfrak{M}$, the notation $A *_{\mathfrak{M}} B$ stands for the free product of $A$ and $B$ in $\mathfrak{M}$.

If $A \in \mathfrak{M}$ then every $\Psi \in A * \mathfrak{M} \mathfrak{M}(x)$ may be interpreted as an $A$-valued function on $A$. Moreover, for every extension $\bar{A}$ of $A, \bar{A} \in \mathfrak{M}, \Psi(x)$ is an $\bar{A}$-valued function on $\bar{A}$. An equation of the form $\Psi(x)=0$ is solvable over $A$ if there exists an extension $\bar{A} \in \mathfrak{M}$ of $A$ such that the equation has a solution in $\bar{A}$. If such a solution can be found in $A$ itself then $\Psi(x)=0$ is said to be solvable in $A$.

Recall the common definition (see, e.g., $[6,17]$ ): an algebra $A$ is (existentially) algebraically closed if every system of equations which is solvable over $A$ is solvable in $A$. Let us restrict this 
definition to a particular case of one equation: we will say $A \in \mathfrak{M}$ to be existentially closed in $\mathfrak{M}$ if every equation $\Psi(x)=0$, where $\Psi(x) \in A *_{\mathfrak{M}} \mathfrak{M}(x)$, which is solvable in an appropriate extension $\bar{A}$ of $A, \bar{A} \in \mathfrak{M}$, has a solution in $A$. This definition is important for model theory, and it can be an efficient tool for studying algebras provided the principal question on the solvability of a particular equation is solved.

A stronger property (see [1]) can be stated as follows: an algebra $A \in \mathfrak{M}$ is called algebraically closed in $\mathfrak{M}$ if for every $\Psi \in A * \mathfrak{M} \mathfrak{M}(x), \Psi \notin A$, the equation $\Psi(x)=0$ is solvable in $A$. We are going to propose an intermediate definition which is sufficient for our purpose.

Definition 1. An algebra $A \in \mathfrak{M}$ is called conditionally closed in $\mathfrak{M}$ if for every $\Psi(x) \in$ $A * \mathfrak{M} \mathfrak{M}(x)$ which is not a constant function on $A$ the equation $\Psi(x)=0$ is solvable in $A$.

Every algebraically closed in $\mathfrak{M}$ algebra is conditionally closed in $\mathfrak{M}$. However, there is plenty of conditionally closed systems that are not algebraically closed in $\mathfrak{M}$. For example, an algebraically closed field is conditionally closed but not algebraically closed in the variety of all associative algebras. Similarly, such a field may be considered as a Poisson algebra with respect to trivial bracket, and the Poisson algebra obtained is conditionally closed but not algebraically closed in the variety of all Poisson algebras.

It is also interesting to compare conditionally closed and existentially closed algebras. Neither of these notions is a formal generalization of another. For example, let $\mathfrak{M}=$ As, the variety of associative algebras, and let $A \in$ As be the algebraic closure of the field $\mathbb{k}(t)$. As an algebraically closed field, $A$ is conditionally closed in As, but the equation $[t, x]=1$ has no solution in $A$ although it is solvable in an appropriate extension (e.g., in the Makar-Limanov's skew field [12]). On the other hand, for the same variety As, the existential algebraic closure (see, e.g., [4, Chapter III]) of quaternions $\mathbb{H}$ is not conditionally closed: equation $i x-x i=1$ has no solution in any extension of $\mathbb{H}$ in As.

Suppose $\mathfrak{M}_{1}$ and $\mathfrak{M}_{2}$ are two varieties of algebras over a field $\mathbb{k}$, and let $\omega: \mathfrak{M}_{1} \rightarrow \mathfrak{M}_{2}$ be a functor which acts as follows: given $A \in \mathfrak{M}_{1}, A^{(\omega)} \in \mathfrak{M}_{2}$ is the same linear space equipped with new operations expressed in terms of initial operations. For example, one may consider the classical functor from the variety of associative algebras into the variety of Lie algebras defined by $[x, y]=x y-y x$.

Another important example comes from the following settings. Let $\mathfrak{M}_{1}=\operatorname{Dif}_{2 n}$ be the variety of commutative associative algebras with $2 n$ pairwise commuting derivations $\partial_{i}, \partial_{i}^{\prime}, i=1, \ldots, n$. Then, given $A \in \operatorname{Dif}_{2 n}$, the same space equipped with new binary operation

$$
\{a, b\}=\sum_{i=1}^{n} \partial_{i}(a) \partial_{i}^{\prime}(b)-\partial_{i}(b) \partial_{i}^{\prime}(a), \quad a, b \in A,
$$

is known to be a Poisson algebra denoted by $A^{(\partial)}$. If we allow the derivations $\partial_{i}, \partial_{i}^{\prime}$ to be non-commuting then (1) defines on a commutative algebra $A$ a structure of a GP-algebra.

In general, $\omega$ may be a functor induced by a morphism of the governing operads. Functors of this kind were closely studied in [14].

Proposition 1. If an algebra $A \in \mathfrak{M}_{1}$ is conditionally closed in $\mathfrak{M}_{1}$ then $A^{(\omega)}$ is conditionally closed in $\mathfrak{M}_{2}$.

Note that for algebraically closed algebras this statement does not hold.

Proof. Since $\omega$ is a functor, the universal property of the free product implies the existence of a homomorphism $\varphi: A^{(\omega)} * \mathfrak{M}_{2} \mathfrak{M}_{2}(x) \rightarrow\left(A *_{\mathfrak{M}_{1}} \mathfrak{M}_{1}(x)\right)^{(\omega)}$ such that $f(a)=\varphi(f)(a)$ for all $f=f(x) \in A^{(\omega)} *_{\mathfrak{M}_{2}} \mathfrak{M}_{2}(x), a \in A^{(\omega)}$.

Therefore, $f$ is not a constant function on $A^{(\omega)}$ if and only if $\varphi(f)$ is not a constant function on $A$. If $A$ is conditionally closed then there exists $a \in A$ such that $\varphi(f)(a)=0$ and thus $f(a)=0$. 
In some cases, the converse statement is true: if $A^{(\omega)}$ is conditionally closed in $\mathfrak{M}_{2}$ then so is $A$ in $\mathfrak{M}_{1}$.

Remark 1. Let $\omega: \mathfrak{M}_{1} \rightarrow \mathfrak{M}_{2}$ be a functor with the following property: if $A \in \mathfrak{M}_{1}$ and $A^{(\omega)}$ is a subalgebra of $C \in \mathfrak{M}_{2}$ then there exists $B \in \mathfrak{M}_{1}$ such that $C=B^{(\omega)}$ and $A$ is a subalgebra of $B$. Then $A$ is conditionally closed in $\mathfrak{M}_{1}$ provided that $A^{(\omega)}$ is conditionally closed in $\mathfrak{M}_{2}$.

Indeed, $A^{(\omega)} * \mathfrak{M}_{2} \mathfrak{M}_{2}(x)$ is a $\mathfrak{M}_{2}$-algebra which contains $A^{(\omega)}$. Hence, $A^{(\omega)} * \mathfrak{M}_{2} \mathfrak{M}_{2}(x)=B^{(\omega)}$ for a $\mathfrak{M}_{1}$-algebra $B$. Therefore, there exists a homomorphism of $\mathfrak{M}_{1}$-algebras $\psi: A * \mathfrak{M}_{1} \mathfrak{M}_{1}(x)$ $\rightarrow B$ such that $\psi(a)=a$ for $a \in A, \psi(x)=x$. Hence, $\varphi(\psi(f))=f$ for all $f \in A *_{\mathfrak{M}_{1}} \mathfrak{M}_{1}(x)$, where $\varphi$ is the homomorphism in the proof of Proposition 1.

Suppose $A^{(\omega)}$ is conditionally closed in $\mathfrak{M}_{2}$. If $f \in A * \mathfrak{M}_{1} \mathfrak{M}_{1}(x)$ is not a constant function on $A$ then so is $g=\psi(f)$ since $\varphi(g(a))=\varphi(g)(a)=f(a)$ for all $a \in A$. Hence, there exists a solution of the equation $g(x)=0$ in $A^{(\omega)}$ which is obviously a solution of $f(x)=0$ in $A$.

The Freiheitssatz problem for a variety $\mathfrak{M}$ is to determine whether every nontrivial equation over the free algebra $\mathfrak{M}(X), X=\left\{x_{1}, x_{2}, \ldots\right\}$, is solvable over $\mathfrak{M}(X)$.

It is obviously equivalent to the following question about free algebras: is the intersection of the ideal $(f)$ generated by an element $f \in \mathfrak{M}(X \cup\{x\})$ and the subalgebra $\mathfrak{M}(X) \subset \mathfrak{M}(X \cup\{x\})$ trivial if $f \notin \mathfrak{M}(X)$ (i.e., depends on $x)$ ? If the answer is positive for all such $f$ then we say that the Freiheitssatz holds for $\mathfrak{M}$.

It is easy to note that if the Freiheitssatz holds of a variety $\mathfrak{M}$ over algebraically closed fields then it holds for $\mathfrak{M}$ over an arbitrary field of the same characteristic.

Lemma 1. Suppose $\mathfrak{M}$ is a variety of algebras with at least one binary operation $\cdot$ in the signature such that $\mathfrak{M}(X)=\mathfrak{M}\left(x_{1}, x_{2}, \ldots\right)$ has no zero divisors with respect to $\cdot$. Then, if for every nonzero polynomial $h=h\left(x_{1}, \ldots, x_{n}\right) \in \mathfrak{M}(X)$ there exists a conditionally closed algebra $A \in \mathfrak{M}$ which does not satisfy the polynomial identity $h\left(x_{1}, \ldots, x_{n}\right)=0$ then the Freiheitssatz holds for $\mathfrak{M}$.

Proof. Suppose $X=\left\{x_{1}, x_{2}, \ldots\right\}, x \notin X$, and let $f=f\left(x, x_{1}, \ldots, x_{n}\right) \in \mathfrak{M}(X \cup\{x\}) \backslash \mathfrak{M}(X)$. Then $f=f_{1}+f_{0}$, where $f_{1}$ belongs to the ideal generated by $x, f_{0} \in \mathfrak{M}(X)$.

Assume $g \in(f) \cap \mathfrak{M}(X), g \neq 0$. Then $h=f_{1} \cdot g \neq 0$, hence, there exist a conditionally closed $A \in \mathfrak{M}$ such that $h\left(x, x_{1}, \ldots, x_{n}\right)$ is not a polynomial identity on $A$. Therefore, there exist $a, a_{1}, \ldots, a_{n} \in A$ such that $f_{1}\left(a, a_{1}, \ldots, a_{n}\right) g\left(a_{1}, \ldots, a_{n}\right) \neq 0$ in $A$, so $f_{1}\left(a, a_{1}, \ldots, a_{n}\right) \neq 0$. On the other hand, $f_{1}\left(0, a_{1}, \ldots, a_{n}\right)=0$. Therefore, $\Psi(x)=f_{1}\left(x, a_{1}, \ldots, a_{n}\right)$ is a non-constant function on $A$. Since $A$ is conditionally closed, there exists $a \in A$ such that $\Psi(a)=f_{1}\left(a, a_{1}, \ldots, a_{n}\right)=$ $-f_{0}\left(a_{1}, \ldots, a_{n}\right)$. Thus, $f\left(a, a_{1}, \ldots, a_{n}\right)=0$ but $g\left(a_{1}, \ldots, a_{n}\right) \neq 0$ which is impossible if $g \in$ $(f) \triangleleft \mathfrak{M}(X \cup\{x\})$.

There is a well-known functor $\omega$ from the variety As of associative algebras to the variety Jord of Jordan algebras: every associative algebra $A$ turns into a Jordan algebra denoted by $A^{(+)}$ under new product $x \circ y=x y+y x$. A Jordan algebra is said to be special if it can be embedded into an algebra of the form $A^{(+)}, A \in$ As. The class of all special Jordan algebras is not a variety since a homomorphic image of a special Jordan algebra may not be special. However, the class of all homomorphic images of all special Jordan algebras is a variety denoted by SJ. The free algebra $\operatorname{SJ}(X)$ is obviously the subalgebra of $\operatorname{As}(X)^{(+)}$generated by $X$ with respect to Jordan product.

Corollary 1. The Freiheitssatz holds for the variety generated by special Jordan algebras over a field of characteristic zero. 
Proof. Let us recall the main features of the construction of the algebraically closed associative noncommutative algebra from [12]. Consider the associative commutative algebra $C$ generated by all rational powers of countably many formal variables $p_{i}, q_{i}, i \in \mathbb{N}$ :

$$
C=\mathbb{k}\left[p_{i}^{\lambda}, q_{i}^{\mu} \mid \lambda, \mu \in \mathbb{Q}, i=1,2, \ldots\right] .
$$

The algebra $C$ has two derivations $\partial_{p}$ and $\partial_{q}$ defined by inductive relations

$$
\begin{aligned}
& \partial_{p}\left(p_{n+1}\right)=p_{n}^{-1} \partial_{p}\left(p_{n}\right), \quad \partial_{p}\left(q_{n+1}\right)=p_{n}^{-1} \partial_{p}\left(p_{n}\right)+q_{n}^{-1} \partial_{p}\left(q_{n}\right), \\
& \partial_{p}\left(q_{n+1}\right)=0, \quad \partial_{q}\left(q_{n+1}\right)=q_{n}^{-1} \partial_{q}\left(q_{n}\right),
\end{aligned}
$$

assuming $\partial_{p}\left(p_{1}\right)=\partial_{q}\left(q_{1}\right)=1, \partial_{p}\left(q_{1}\right)=\partial_{q}\left(p_{1}\right)=0$.

The set of all monomials in $C$ is an Abelian group which is linearly ordered by means of

$$
p_{1} \ll q_{1} \ll p_{2} \ll q_{2} \ll \cdots<1,
$$

where $a \ll b$ means $a^{\lambda}<b$ for all $\lambda>0$. Let us denote this group by $G$, and let $G_{n, m}$ stand for the subgroup of $G$ generated by $p_{i}^{\lambda}, q_{i}^{\mu}, i=1, \ldots, n, \lambda, \mu \in \frac{1}{m} \mathbb{Z}$.

A Malcev-Newmann series over $G$ is a transfinite formal series

$$
a=\sum_{g \in G} a(g) g, \quad a(g) \in \mathbb{k},
$$

in which the set $\operatorname{supp}(a)=\{g \in G \mid a(g) \neq 0\}$ is a well-ordered subset of $G$.

Denote by $A_{n, m}$ the set of all Malcev-Newmann series $a$ over $G$ with $\operatorname{supp}(a) \subset G_{n, m}$, $n, m \in \mathbb{N}$. The union $A$ of all $A_{n, m}$ is an associative and commutative subalgebra in the algebra of all Malcev-Newmann series over $G$. Derivations $\partial_{p}$ and $\partial_{q}$ may be naturally expanded to derivations of $A$.

Finally, define a new binary operation $*$ on the space $A$ :

$$
a * b=\sum_{n=0}^{\infty} \frac{1}{n !} \partial_{q}^{n}(a) \partial_{p}^{n}(b) .
$$

It is proved in [12] that $A$ with respect to $*$ is an algebraically closed skew field. Note that

$$
q_{1} * p_{1}-p_{1} * q_{1}=1
$$

so $A$ contains the first Weyl algebra $W_{1}$. Since $A$ is a skew field, it contains the skew field of fractions $Q\left(W_{1}\right)$, which contains a two-generated free associative algebra [11]. Thus, $A$ contains free associative algebra in any finite number of generators $x_{1}, \ldots, x_{n}$. The special Jordan algebra $A^{(+)}$is conditionally closed by Proposition 1 and contains free special Jordan algebra $\mathrm{SJ}\left(x_{1}, \ldots, x_{n}\right)$. Therefore, the variety SJ satisfies all conditions of Lemma 1.

Note that for the entire variety Jord the Freiheitssatz does not hold [14].

\section{Jacobian polynomials in free anti-commutative algebras}

In order to prove the Freiheitssatz for a variety $\mathfrak{M}$ by means of Lemma 1, we have to construct an algebra in $\mathfrak{M}$ which is conditionally closed and does not satisfy a given polynomial identity.

In this section, we discuss technical questions that are used in subsequent sections for the study of polynomial identities on generic Poisson algebras. 


\subsection{Preliminaries on $\mathrm{AC}(\boldsymbol{X})$}

Let $X$ be a set of generators, and let $X^{*}$ stand for the set of all (nonempty) associative words $u$ in the alphabet $X$. Denote by $X^{* *}$ the set of all non-associative words in $X$. Given a word $u \in X^{*}$, denote by $(u)$ a non-associative word obtained from $u$ by some bracketing. We will also use $\left[X^{*}\right]$ to denote the set of all associative and commutative words in $X$. Given $u \in X^{*}$, $[u]$ stands for the commutative image of $u$.

Suppose $X^{* *}$ is equipped with a linear order $\preceq$. A non-associative word $u \in X^{* *}$ is normal if either $u=x \in X$ or $u=u_{1} u_{2}$, where $u_{1}$ and $u_{2}$ are normal and $u_{1} \prec u_{2}$. Obviously, normal words in $X^{* *}$ form a linear basis of the free anti-commutative algebra $\operatorname{AC}(X)$ generated by $X$ (see $[2,21])$.

Let us call the elements of $\mathrm{AC}(X)$ AC-polynomials. Given $u \in X^{* *}$, define $\operatorname{deg} u$ to be the length of $u$. Thus, we have a well-defined degree function on $\mathrm{AC}(X)$.

Choose a generator $x_{i} \in X=\left\{x_{1}, \ldots, x_{n}\right\}$ and denote by $V_{i}$ the subspace of $\mathrm{AC}(X)$ spanned by all nonassociative words linear in $x_{i}$. Fix a linear order $\preceq$ on $X^{* *}$ such that any nonassociative word which contains $x_{i}$ is greater than any word without $x_{i}$ (there exist many linear orders with this property). With respect to such an order, the unique normal form of a monomial $w \in V_{i}$ is

$$
w=\left\{u_{1},\left\{u_{2}, \ldots\left\{u_{k}, x_{i}\right\} \ldots\right\}\right\}
$$

where $u_{j}, j=1, \ldots, k$, are normal words in the alphabet $X \backslash\left\{x_{i}\right\}$. The number $k$ is called $x_{i}$-height [5] of $w$, let us denote it by ht $\left(w, x_{i}\right)$.

Let $V_{0}=\bigcap_{i=1}^{n} V_{i}$ be the space of polylinear AC-polynomials. It is easy to compute $x_{i}$-height of a nonassociative word $w \in V_{0}$ just by the number of brackets in $w$ to the left of $x_{i}$, assuming $\{$ is counted as 1 and $\}$ as -1 . For example, the $x_{4}$-height of $\left\{\left\{x_{1},\left\{\left\{x_{2}, x_{3}\right\}, x_{4}\right\}\right\},\left\{x_{5}, x_{6}\right\}\right\}$ is equal to 3 .

Denote by $M(\mathrm{AC}(X))$ the algebra of left multiplications on $\mathrm{AC}(X)$, i.e., the subalgebra of End $_{\mathbb{k}} \mathrm{AC}(X)$ generated by

$$
\text { ad } g: f \mapsto\{g, f\}, \quad f, g \in \mathrm{AC}(X)
$$

Since the variety $\mathrm{AC}$ of anti-commutative algebras is a Schreier one, $M(\mathrm{AC}(X))$ is a free associative algebra (see [22]). Let $U$ stand for the set of all normal words in $X^{* *}$. It is easy to see that $M(\mathrm{AC}(X)) \simeq \operatorname{As}(U)$ provided that we identify ad $u$ with $u \in U$.

Denote by $U_{i}$ the set of normal words in the alphabet $X \backslash\left\{x_{i}\right\}$. Then $V_{i}$ is a 1-generated free left module over $\operatorname{As}\left(U_{i}\right)$ : every word of the form (2) may be uniquely presented as

$$
w=W\left(x_{i}\right), \quad W=\operatorname{ad} u_{1} \operatorname{ad} u_{2} \cdots \operatorname{ad} u_{k},
$$

where $u_{1}, \ldots, u_{k} \in U_{i}$.

Denote by $*$ the involution of $\operatorname{As}\left(U_{i}\right)$ given by $\left(u_{1} \cdots u_{k}\right)^{*}=(-1)^{k} u_{k} \cdots u_{1}, u_{j} \in U_{i}$.

Definition 2. A linear transformation of $V_{i}$ defined by the rule

$$
F_{i}: W\left(x_{i}\right) \mapsto-W^{*}\left(x_{i}\right)
$$

is called an $x_{i}$-flip. Obviously, $\left(F_{i}\right)^{-1}=F_{i}$.

The set of all flips $\left\{F_{1}, \ldots, F_{n}\right\}$ acts on the space $V_{0}$ and thus generates a group $\mathcal{F} \subseteq \operatorname{GL}\left(V_{0}\right)$. Given a normal word $u \in V_{0}$, the orbit $\mathcal{F} u$ consists of AC-monomials (polynomials of the form $\varepsilon v$, $v$ is a nonassociative word, $\varepsilon= \pm 1$ ). 
Lemma 2. Let $w=\left\{x_{1},\left\{x_{2}, \ldots\left\{x_{n-1}, x_{n}\right\} \ldots\right\}\right\} \in V_{0}$. Then

$$
(-1)^{\sigma}\left\{x_{1 \sigma},\left\{x_{2 \sigma}, \ldots\left\{x_{(n-1) \sigma}, x_{n \sigma}\right\} \ldots\right\}\right\} \in \mathcal{F} w
$$

for every $\sigma \in S_{n}$ (here $(-1)^{\sigma}$ stands for the parity of a permutation $\sigma$ ).

Proof. In order to apply $F_{i}$ to the word $w$ we have to rewrite it in the form (2) by means of anticommutativity and then invert the order of $u_{j}$ s. Let us denote $u=\left\{x_{i+1}, \ldots\left\{x_{n-1}, x_{n}\right\} \ldots\right\}$. Then

$$
\begin{aligned}
& w=\left\{x_{1}, \ldots, x_{i-1},\left\{x_{i},\left\{x_{i+1}, \ldots\left\{x_{n-1}, x_{n}\right\} \ldots\right\}\right\} \ldots\right\} \\
& \quad=\left\{x_{1}, \ldots, x_{i-1},\left\{x_{i}, u\right\} \ldots\right\}=-\left\{x_{1}, \ldots, x_{i-1},\left\{u, x_{i}\right\} \ldots\right\}, \\
& F_{i} w=-(-1)^{i}\left\{u,\left\{x_{i-1}, \ldots,\left\{x_{1}, x_{i}\right\} \ldots\right\}\right\}=(-1)^{i}\left\{u,\left\{x_{i-1}, \ldots,\left\{x_{2},\left\{x_{i}, x_{1}\right\}\right\} \ldots\right\}\right\} .
\end{aligned}
$$

The expression for $F_{i} w \in \pm V_{1}$ is already in the form (2). In the same way, apply $F_{1}$ to $F_{i} w$ :

$$
F_{1} F_{i} w=(-1)^{i} F_{1}\left\{u,\left\{x_{i-1}, \ldots,\left\{x_{i}, x_{1}\right\} \ldots\right\}\right\}=\left\{x_{i},\left\{x_{2}, \ldots,\left\{x_{i-1},\left\{u, x_{1}\right\} \ldots\right\}\right\} .\right.
$$

It remains to apply anti-commutativity to write the result $F_{1} F_{i} w$ in the form (2):

$$
F_{1} F_{i} w=-\left\{x_{i},\left\{x_{2}, \ldots x_{i-1},\left\{x_{1},\left\{x_{i+1}, \ldots\left\{x_{n-1}, x_{n}\right\} \ldots\right\}\right\}\right\}\right\},
$$

$i=2, \ldots, n$. Since transpositions of the form $(1 i)$ generate the entire symmetric group $S_{n}$, the lemma is proved.

\subsection{Jacobian AC-polynomials}

In this section, we describe polylinear AC-polynomials that have a specific property if considered as elements of the free GP-algebra.

Suppose $\Psi\left(x_{1}, \ldots, x_{n}\right)$ is an element of the free GP-algebra $\operatorname{GP}(X), X=\left\{x_{1}, \ldots, x_{n}\right\}$ which is linear with respect to $x_{n}$. We will say that $\Psi$ is a derivation with respect to $x_{n}$ if

$$
\Psi\left(x_{1}, \ldots, x_{n-1}, y z\right)=y \Psi\left(x_{1}, \ldots, x_{n-1}, z\right)+z \Psi\left(x_{1}, \ldots, x_{n-1}, y\right)
$$

in the free GP-algebra $\operatorname{GP}\left(x_{1}, \ldots, x_{n-1}, y, z\right)$.

Definition 3. A polylinear AC-polynomial $\Psi=\Psi\left(x_{1}, \ldots, x_{n}\right) \in V_{0}$ is said to be a Jacobian if $\Psi$ is a derivation with respect to each variable $x_{i}, i=1, \ldots, n$. A polylinear element of $\operatorname{GP}(X)$ with the same property is called a Jacobian GP-polynomial.

For free Lie algebra considered as a part of the free Poisson algebra, a similar notion was considered in [5]. Obviously, if $n=2$ then $C_{2}=\left\{x_{1}, x_{2}\right\}$ is a Jacobian AC-polynomial. It was shown in [5] that there are no other Jacobian Lie polynomials (up to a multiplicative constant). However, there exists a Jacobian AC-polynomial of degree 3:

$$
J_{3}=\left\{\left\{x_{1}, x_{2}\right\}, x_{3}\right\}+\left\{\left\{x_{2}, x_{3}\right\}, x_{1}\right\}+\left\{\left\{x_{3}, x_{1}\right\}, x_{2}\right\} .
$$

The main purpose of this section is to show that $C_{2}$ and $J_{3}$ exhaust all Jacobian AC-polynomials.

For a generic Poisson algebra $A, a \in A$, consider the linear map ad $a: x \mapsto\{a, x\}, x \in A$. The set of all such transformations $\{\operatorname{ad} a \mid a \in A\} \subset \operatorname{End}_{\mathbb{k}}(A)$ generates a Lie subalgebra $L(A) \subset$ $\operatorname{gl}(A)=\operatorname{End}_{\mathbb{k}}(A)^{(-)}$.

Given $L \in L\left(\operatorname{GP}\left(x_{1}, \ldots, x_{n-1}\right)\right)$, one may easily note that $L\left(x_{n}\right) \in \operatorname{GP}\left(x_{1}, \ldots, x_{n}\right)$ is a derivation with respect to $x_{n}$. Indeed, the Leibniz identity implies that ad $u, u \in \operatorname{GP}\left(x_{1}, \ldots, x_{n-1}\right)$, is a derivation with respect to $x_{n}$, and the commutator of derivations is a derivation itself. 
Lemma 3. Let $\Psi\left(x_{1}, \ldots, x_{n}\right) \in \mathrm{AC}(X) \subset \mathrm{GP}(X)$ be a polylinear element such that $\Psi$ is a derivation with respect to $x_{n}$. Then there exists $L \in L(\mathrm{AC}(X))$ such that $\Psi=L\left(x_{n}\right)$.

Proof. The algebra of multiplications $M\left(\mathrm{AC}\left(x_{1}, \ldots, x_{n-1}\right)\right) \simeq \operatorname{As}\left(U_{n}\right)$ contains free Lie subalgebra $\mathcal{L}=\operatorname{Lie}\left(U_{n}\right) \subset \operatorname{As}\left(U_{n}\right)^{(-)}$generated by ad $u$ for all normal words $u \in U_{n}$.

As $\mathcal{L}$ acts on $V=\mathrm{AC}\left(x_{1}, \ldots, x_{n-1}, y, z\right)$, we have the standard $\mathcal{L}$-module structure on $V \otimes V$, given by

$$
a(u \otimes v)=a u \otimes v+u \otimes a v, \quad a \in \mathcal{L}, \quad u, v \in V .
$$

Since $\operatorname{As}\left(U_{n}\right)=U(\mathcal{L})$ is the universal enveloping algebra of $\mathcal{L}, V \otimes V$ is also an $U(\mathcal{L})$-module given by

$$
a(u \otimes v)=\sum_{(a)} a_{(1)} u \otimes a_{(2)} v, \quad a \in U(\mathcal{L}), \quad u, v \in V
$$

where $\Delta: a \mapsto \sum_{(a)} a_{(1)} \otimes a_{(2)}$ is the standard coproduct in $U(\mathcal{L})$.

An AC-polynomial $\Psi\left(x_{1}, \ldots, x_{n}\right)$ may be presented as $L\left(x_{n}\right)$ for some $L \in \operatorname{As}\left(U_{n}\right)$. By definition, $\Psi$ is a derivation with respect to $x_{n}$ if and only if

$$
L(u \otimes v)=L(u) \otimes v+u \otimes L(v) \in V \otimes V
$$

for all $u, v \in V$. Since $V \otimes V$ is a faithful $U(\mathcal{L})$-module, we obtain $\Delta(L)=L \otimes 1+1 \otimes L$, thus the Friedrichs criterion for the Lie elements in $\operatorname{As}\left(U_{n}\right)$ implies $L$ to be an element of $\mathcal{L}$, which proves the claim.

Define a linear map

$$
D\left(\cdot, x_{n} ; y, z\right): V_{0} \rightarrow \mathrm{GP}\left(x_{1}, \ldots, x_{n-1}, y, z\right)
$$

as follows: given $w=W\left(x_{n}\right), W \in M\left(\mathrm{AC}\left(x_{1}, \ldots, x_{n-1}\right)\right)$, set

$$
D\left(w, x_{n} ; y, z\right)=W(y z)-y W(z)-z W(y) .
$$

A polylinear AC-polynomial $\Psi\left(x_{1}, \ldots, x_{n}\right) \in V_{0}$ is a derivation with respect to $x_{n}$ if and only if $D\left(\Psi, x_{n} ; y, z\right)=0$, or, as we have noticed above, $W$ is primitive $(\Delta(W)=1 \otimes W+W \otimes 1)$. This property of $W$ is homogeneous in $\operatorname{As}\left(U_{n}\right)$, i.e., $W$ splits into groups of homogeneous summands $W=W_{1}+\cdots+W_{l}$, where $W_{i}\left(x_{n}\right)$ is again a derivation with respect to $x_{n}$.

Remark 2. Suppose $\Psi=L\left(x_{n}\right) \in \mathrm{AC}(X)$ is a polynomial as in Lemma 3. Then the operator $L$ belongs to $\operatorname{Lie}\left(\operatorname{ad} u_{1}, \ldots\right.$, ad $\left.u_{k}\right)$ for some normal words $u_{1}, \ldots, u_{k} \in U_{n}$ (assume $k$ is minimal). For every $i=1, \ldots, k \Psi$ must contain a term

$$
\left\{u_{i_{1}},\left\{u_{i_{2}} \ldots\left\{u_{i}, x_{n}\right\} \ldots\right\}\right\}
$$

in which the word $u_{i}$ appears as the last entry.

Without loss of generality, assume $L$ is polylinear with respect to $\operatorname{ad} u_{1}, \ldots$, ad $u_{k}$. As an element of the free Lie algebra it may be uniquely written as a linear combination of $w_{i_{1}, \ldots, i_{k-1}}=$ $\left[\operatorname{ad} u_{i_{1}},\left[\operatorname{ad} u_{i_{2}}, \ldots,\left[\operatorname{ad} u_{i_{k-1}}, \operatorname{ad} u_{i}\right] \ldots\right]\right]$. The expansion of such a monomial in the free associative algebra $\operatorname{As}\left(U_{n}\right)$ contains unique term ad $u_{i_{1}}$ ad $u_{i_{2}} \ldots$ ad $u_{i_{k-1}}$ ad $u_{i} \in \operatorname{As}\left(U_{n}\right)$ ending with ad $u_{i}$. These terms for different $w_{i_{1}, \ldots, i_{k-1}}$ do not cancel. 
Corollary 2. Let $\Psi\left(x_{1}, \ldots, x_{n}\right) \in \mathrm{AC}(X)$ be a polylinear AC-polynomial of degree $n$ such that $\Psi$ is a derivation with respect to $x_{n}$. Suppose $\Psi=\sum_{w} \alpha_{w} w, w \in X^{* *}$ are normal words, and

$$
\max _{w: \alpha_{w} \neq 0} \operatorname{ht}\left(w, x_{n}\right) \geq \max _{w: \alpha_{w} \neq 0} \operatorname{ht}\left(w, x_{i}\right), \quad i=1, \ldots, n
$$

$\left(x_{n}\right.$ has maximal height in $\left.\Psi\right)$. Then

$$
\max _{w: \alpha_{w} \neq 0} \operatorname{ht}\left(w, x_{n}\right)=n-1
$$

and thus $\Psi$ contains a monomial of the form

$$
w=\left\{x_{1 s}, \ldots\left\{x_{(n-1) s}, x_{n}\right\} \ldots\right\}
$$

for some $s \in S_{n-1}$.

Proof. Assume $k<n-1$ is the maximal height of $x_{n}$ in $\Psi$, i.e., $\Psi$ contains a summand of the form $\left\{u_{1}, \ldots\left\{u_{k}, x_{n}\right\} \ldots\right\}, k<n-1$. Then there exists at least one $u_{i}$ whose degree is greater than 1. Remark 2 implies that $\Psi$ contains a summand $\alpha_{w} w$, where $w=\left\{u_{j_{1}}, \ldots\left\{u_{j_{k}}, x_{n}\right\} \ldots\right\}$, $j_{k}=i, \alpha_{w} \neq 0$. Since ht $\left(u_{i}, x_{j}\right)>1$ for some $x_{j}$, we have $\operatorname{ht}\left(w, x_{j}\right)>k$, which contradicts to the condition ht $\left(w, x_{j}\right) \leq k$. Hence, $k=n-1$.

Lemma 4. Suppose $\Psi=\Psi\left(x_{1}, \ldots, x_{n}\right)$ is a Jacobian AC-polynomial. Then $\Psi$ is invariant with respect to the action of the group $\mathcal{F}$ generated by all $x_{i}$-flips, $i=1, \ldots, n$.

Proof. Let us fix $i \in\{1, \ldots, n\}$. Without loss of generality we may assume $i=n$. By Lemma 3 , $\Psi=L\left(x_{n}\right)$, where $L$ is a linear operator constructed by commutators of operators ad $u, u \in U_{n}$. The set of all such ad $u$ generates an associative subalgebra $\mathcal{U} \subset \operatorname{End}_{\mathbb{k}} V_{n}, \mathcal{U} \simeq \operatorname{As}\left(U_{n}\right)$.

Since $\Psi$ is polylinear (with respect to $X$ ), $L$ naturally splits into a sum of operators presented by polylinear (with respect to $U_{n}$ ) elements of $\mathcal{U}$.

Consider the linear transformation $\tau$ of $\mathcal{U}$ given by

$$
\tau: W \mapsto-W^{*}, \quad W \in \mathcal{U} .
$$

The map $\tau$ acts as an identity on $\operatorname{Lie}\left(U_{n}\right) \subset \mathcal{U}^{(-)}$, it follows from the obvious observation $\tau\left(\left[W_{1}, W_{2}\right]\right)=\left[\tau\left(W_{1}\right), \tau\left(W_{2}\right)\right]$ for $W_{1}, W_{2} \in \mathcal{U}$.

By Definition 2,

$$
F_{n}(\Psi)=F_{n}\left(L\left(x_{n}\right)\right)=\tau(L)\left(x_{n}\right)=L\left(x_{n}\right)=\Psi .
$$

As $\Psi$ is invariant with respect to all flips, we have $\mathcal{F}(\Psi)=\{\Psi\}$.

Lemma 5. Suppose $U=\left\{u_{1}, \ldots, u_{m}\right\}$ is a set, $\operatorname{As}(U)$ is the free associative algebra generated by $U$, Lie $(U)$ is the free Lie algebra generated by $U, \operatorname{Lie}(U) \subset \operatorname{As}(U)^{(-)}$. Consider

$$
A_{m}=\sum_{s \in S_{m}}(-1)^{s} u_{1 s} \cdots u_{m s}
$$

Then $A_{m} \in \operatorname{Lie}(U)$ if and only if $m=1$ or $m=2$.

Proof. For $m=1,2$ it is obvious that $A_{m} \in \operatorname{Lie}(U)$.

Assume $m \geq 3$ and $A_{m} \in \operatorname{Lie}(U)$. Consider the homomorphism $\Phi: \operatorname{As}(U) \rightarrow \wedge(\mathbb{k} U)$ given by $u \mapsto u$, where $\wedge(\mathbb{k} U)$ is the exterior algebra of the linear space spanned by $U$. Note that $\Phi\left(A_{m}\right)=m ! u_{1} \cdots u_{m} \neq 0$ in $\wedge(\mathbb{k} U)$. However, $\Phi(\operatorname{Lie}(U)) \subset \wedge(\mathbb{k} U)^{(-)}$is a Lie subalgebra generated by $U$. It is easy to see that $\wedge(\mathbb{k} U)^{(-)}$is a 3-nilpotent Lie algebra, so $\Phi(\operatorname{Lie}(U))$ does not contain elements of degree $m \geq 3$. 
Theorem 1. Let $X=\left\{x_{1}, \ldots, x_{n}\right\}$, and let $\Psi=\Psi\left(x_{1}, \ldots, x_{n}\right) \in \mathrm{AC}(X)$ be a Jacobian ACpolynomial. Then either $n=2$ and $\Psi=\alpha C_{2}$, or $n=3$ and $\Psi=\alpha J_{3}$, where $\alpha \in \mathbb{k}^{*}$.

Proof. By Lemma $4 F \Psi=\Psi$ for every $F \in \mathcal{F}$. Without loss of generality we may assume that $x_{n}$ has the maximal height in $\Psi$ (re-numerate variables if needed). Corollary 2 implies that $\Psi$ contains a summand of the form $\alpha w$, where $\alpha \in \mathbb{k}^{*}, w=\left\{x_{1 s}, \ldots\left\{x_{(n-1) s}, x_{n}\right\} \ldots\right\}$ for some $s \in S_{n-1}$. Without loss of generality, $\alpha=1$ and $s=$ id. By Lemma 2, $\Psi$ contains all monomials obtained from $w$ by all permutations of variables, i.e.,

$$
\Psi=\sum_{s \in S_{n-1}}(-1)^{s}\left\{x_{1 s}, \ldots\left\{x_{(n-1) s}, x_{n}\right\} \ldots\right\}+\Phi\left(x_{1}, \ldots, x_{n}\right),
$$

where the $x_{n}$-height of all monomials in $\Phi$ is smaller than $n-1$. Since all summands of $\Psi$ with the same $x_{n}$-height form a derivation with respect to $x_{n}$, the AC-polynomial

$$
\Psi_{1}=\sum_{s \in S_{n-1}}(-1)^{s}\left\{x_{1 s}, \ldots\left\{x_{(n-1) s}, x_{n}\right\} \ldots\right\}
$$

must be a derivation with respect to $x_{n}$. But

$$
\Psi_{1}=A_{n-1}\left(u_{1}, \ldots, u_{n-1}\right)\left(x_{n}\right), \quad u_{i}=\operatorname{ad} x_{i},
$$

so $A_{n-1}\left(u_{1}, \ldots, u_{n-1}\right) \in \operatorname{Lie}\left(u_{1}, \ldots, u_{n-1}\right)$. By Lemma $5, n-1 \leq 2$, so $n \leq 3$. Obviously, $C_{2}$ and $J_{3}$ are the only Jacobian AC-polynomials for $n=2$ and $n=3$, respectively.

\section{Identities of generic Poisson algebras}

Let $A$ be a GP-algebra, and let $f \in \operatorname{GP}\left(x_{1}, \ldots, x_{n}\right), f \neq 0$. As usual, we say that $f$ is a polynomial identity on $A$ if for every homomorphism $\varphi: \operatorname{GP}\left(x_{1}, \ldots, x_{n}\right) \rightarrow A$ we have $\varphi(f)=0$. In this case we also say that $A$ satisfies the polynomial identity $f$.

Proposition 2. Suppose a GP-algebra A satisfies a polynomial identity. Then there exists a polynomial identity $\Psi$ on $A$ which is a Jacobian GP-polynomial.

This statement, as well as its proof, is similar to the result by Farkas [5] on polynomial identities of Poisson algebras.

Proof. The standard linearization procedure (see, e.g., [23, Chapter 1]) allows to assume that $A$ satisfies a polylinear polynomial identity $f \in \operatorname{GP}(X), X=\left\{x_{1}, \ldots, x_{n}\right\}$.

As an element of $\mathrm{GP}(X), f$ may be uniquely presented as a linear combination of GPmonomials $w=u_{1} \cdots u_{k}, u_{j} \in U$, where $U \subset \mathrm{AC}(X)$ is the set of normal words. We may assume that $u_{j}$ are of degree two or more (if an AC-monomial of degree one appears, e.g., $u_{j}=x_{i}$, then one may plug in $x_{i}=1$ and obtain a polylinear polynomial identity without $x_{i}$ ). Denote by $\mathrm{FH}_{i}(w)$ (the Farkas height) the degree of $u_{j}$ in which the variable $x_{i}$ occurs, and let $\mathrm{FH}_{i}(f)$ be the maximal of $\mathrm{FH}_{i}(w)$ among all GP-monomials $w$ that appear in $f$ with a nonzero coefficient. Finally, set

$$
\mathrm{FH}(f)=\sum_{i=1}^{n} 3^{\mathrm{FH}_{i}(f)} .
$$

Observe that if $f$ is not a derivation in $x_{i}$ then the derivation difference $D\left(f, x_{i} ; x_{i}, x_{n+1}\right)$ is a nonzero polylinear element of $\operatorname{GP}\left(X \cup\left\{x_{n+1}\right\}\right)$ which has a smaller Farkas height. Indeed, for a GP-monomial $w$ from $f$ we have

$$
\mathrm{FH}_{j}\left(D\left(w, x_{i} ; x_{i}, x_{n+1}\right)\right) \leq \mathrm{FH}_{j}(w)
$$




$$
\begin{aligned}
& \mathrm{FH}_{i}\left(D\left(w, x_{i} ; x_{i}, x_{n+1}\right)\right) \leq \mathrm{FH}_{i}(w)-1, \\
& \mathrm{FH}_{n+1}\left(D\left(w, x_{i} ; x_{i}, x_{n+1}\right)\right) \leq \mathrm{FH}_{i}(w)-1,
\end{aligned}
$$

which implies

$$
\mathrm{FH}(w)-\mathrm{FH}\left(D\left(w, x_{i} ; x_{i}, x_{n+1}\right)\right) \leq 3^{\mathrm{FH}_{i}(w)}-2 \cdot 3^{\mathrm{FH}_{i}(w)-1}>0 .
$$

Obviously, $D\left(f, x_{i} ; x_{i}, x_{n+1}\right)$ is a polynomial identity on $A$.

Therefore, after a finite number of steps we obtain a nonzero polynomial identity on $A$ which is a Jacobian GP-polynomial in a larger set of variables $\widetilde{X} \supseteq X$.

Let us recall the notion of fine grading [5]. First, given a set $X$, the free anti-commutative algebra $\mathrm{AC}(X)$ carries $\left[X^{*}\right]$-grading such that $u \in X^{* *}$ has weight $[u]$. Next, if $w=\left(u_{1}\right) \cdots\left(u_{n}\right) \in$ $\operatorname{GP}(X), u_{i} \in X^{*}$, then the weight of $w$ is $\left[u_{1}\right]+\cdots+\left[u_{n}\right] \in \mathbb{k}\left[X^{*}\right]$. As a result,

$$
\mathrm{GP}(X)=\bigoplus_{p \in \mathbb{Z}_{+}\left[X^{*}\right] \backslash\{0\}} \operatorname{GP}_{p}(X)
$$

where $\mathbb{Z}_{+}$stands for the set of non-negative integers, $\mathrm{GP}_{p}(X)$ is the space spanned by all generic Poisson monomials of degree $p$. An element $f \in \mathrm{GP}_{p}(X)$ is said to be finely homogeneous.

Proposition 3. A Jacobian GP-polynomial $\Psi$ can be presented as a linear combination of products of Jacobian AC-polynomials (on the appropriate set of variables).

Proof. Let $X=\left\{x_{1}, x_{2}, \ldots, x_{n}\right\}$ be a set of variables, and let $U=\left\{u_{1}, u_{2}, \ldots\right\}$ be the set of normal nonassociative words in $X$ (with respect to some ordering), then $\operatorname{GP}(X)=\mathbb{k}[U]$. For $\Psi \in \operatorname{GP}(X)$, denote by $\operatorname{supp}(\Psi)$ all variables from $X$ that appear in $\Psi$ and by $\operatorname{psupp}(\Psi)$ all elements from $U$ that appear in $\Psi$.

Suppose $f \in \operatorname{GP}\left(x_{1}, \ldots, x_{n}\right) \subseteq \operatorname{GP}(X)$ is a Jacobian GP-polynomial. Without loss of generality we may assume $f$ to be finely homogeneous and $f \notin \mathrm{AC}(X)$. Proceed by induction on $|\operatorname{psupp}(f)|$.

Consider a GP-monomial $w$ in $f$. Since $f \notin \mathrm{AC}(X)$, there exist $u_{i}$ and $w^{\prime} \neq 1$ for which $w=u_{i} w^{\prime}$. Write $f=u_{i} g+h, g, h \in \mathrm{GP}(X), g \neq 1$, where all GP-monomials of $h$ are not divisible by $u_{i}$ (in $\mathbb{k}[U]$ ). Since $f$ is polylinear, $\operatorname{supp}(g) \cap \operatorname{supp}\left(u_{i}\right)=\varnothing$.

Denote by $D_{i}$ a map $\operatorname{GP}(X) \rightarrow \operatorname{GP}(X \cup\{y, z\})$ defined as follows:

$$
D_{i}(\Psi)= \begin{cases}D\left(\Psi, x_{i} ; y, z\right), & x_{i} \in \operatorname{supp}(\Psi), \\ \Psi, & x_{i} \notin \operatorname{supp}(\Psi) .\end{cases}
$$

Then $D_{j}(f)=u_{i} D_{j}(g)+D_{j}(h)=0$ if $x_{j} \in \operatorname{supp}(g)$.

Consider $\operatorname{GP}(X \cup\{y, z\})$ as a polynomial algebra with a set $\widetilde{U}$ of generators including $U$. Then $u_{i} \notin \operatorname{psupp}(h)$ and $u_{i} \notin \operatorname{psupp}\left(D_{j}(h)\right)$. Hence, $D_{j}(g)=0$ and $g$ is a Jacobian GP-polynomial.

Let us now fix the deg-lex order on the set $\left[U^{*}\right]$, i.e., commutative monomials in $U$ are first compared by their length and then lexicographically, assuming $u_{1}<u_{2}<\cdots$. Recall that $f=u_{i} g+h$, where $\operatorname{psupp}(h) \not \supset u_{i}$, and presented $h$ as $h=g p+r$, where all GP-monomials of $r$ are not divisible (in $\mathbb{k}[U]$ ) by the leading GP-monomial $\bar{g}$ of $g$. Then $f=g q+r, q=u_{i}+p$, and $\operatorname{psupp}(r) \not \supset u_{i}$. In particular, $\operatorname{psupp}(r) \subset \operatorname{psupp}(f)$.

By definition, $D_{j}(f)=g D_{j}(q)+D_{j}(r)=0$ if $x_{j} \in \operatorname{supp}(q)$. If $D_{j}(q) \neq 0$ then some of the monomials in $D_{j}(r)$ are divisible by $\bar{g}$. Consider a GP-monomial $M$ of $r$. Since it is not divisible by $\bar{g}$ there is at least one variable $u_{a}$ which appears in $\bar{g}$ and does not appear in $M$. Note that if $\operatorname{supp}\left(u_{b}\right) \not \supset x_{i}$ then $D_{i}\left(u_{b}\right)=u_{b}$, and if $\operatorname{supp} u_{b} \ni x_{i}$ then $D_{i}\left(u_{b}\right)$ is a GP-polynomial of degree two (in $\mathbb{k}[\widetilde{U}]$ ) in which none of variables belongs to $U$. Hence, $D_{j}(M)$ is not divisible by $u_{a}$ and 
none of the GP-monomials of $D_{j}(r)$ is divisible by $\bar{g}$. Therefore, $D_{j}(q)=0$ and $q$ is a Jacobian GP-polynomial.

Since a product of two Jacobian GP-polynomials is also Jacobian (with respect to the corresponding sets of variables), $r=f-g q$ is a Jacobian GP-polynomial. By induction, the statement holds for $r$, as well as for $g$ and $q$.

Corollary 3. Let $F\left(t_{1}, \ldots, t_{n}\right) \in \mathrm{GP}\left(t_{1}, t_{2}, \ldots\right)$ be a finely homogeneous Jacobian GP-polynomial. Then $F$ contains a summand $\alpha u_{1} \cdots u_{k}$, where $\alpha \in \mathbb{k}^{*}, u_{i} \in \mathrm{AC}\left(t_{1}, t_{2}, \ldots\right)$ are of the form

$$
\left\{t_{i_{1}}, t_{i_{2}}\right\} \quad \text { or } \quad\left\{t_{i_{1}},\left\{t_{i_{2}}, t_{i_{3}}\right\}\right\} .
$$

\section{The Freiheitssatz for (generic) Poisson algebras}

The following statement is well-known in the theory of differential fields $[7,15]$. We will sketch a proof below in order to make the exposition more convenient for a reader. Recall that the characteristic of the base field $\mathbb{k}$ is assumed to be zero, and that $\operatorname{Dif}_{n}$ denotes the variety of commutative associative algebras with $n$ pairwise commuting derivations.

Theorem 2. Every algebra from Dif $_{n}$ which is a field can be embedded into an algebraically closed algebra in Dif $_{n}$.

Proof. Let $F$ be a differential field of characteristic zero with a set $\Delta=\left\{\partial_{i} \mid i=1, \ldots, n\right\}$ of pairwise commuting derivations. Denote by $F[x ; \Delta]=F *$ Dif $_{n} \operatorname{Dif}_{n}(x)$ the set of all differential polynomials in one variable $x$ over $F$. Suppose $f(x) \in F[x ; \Delta] \backslash F$. Then there exists a differential field $K$ which is an extension of the differential field $F$ such that the equation $f(x)=0$ has a solution in $K$.

Indeed, differential polynomials $F[x ; \Delta]$ may be considered as ordinary polynomials in infinitely many variables

$$
X=\left\{x^{\left(i_{1}, \ldots, i_{n}\right)} \mid\left(i_{1}, \ldots, i_{n}\right) \in \mathbb{Z}_{+}^{n}\right\},
$$

where $x^{\left(i_{1}, \ldots, i_{n}\right)}$ is identified with $\partial_{1}^{i_{1}} \cdots \partial_{n}^{i_{n}}(x)$. Then the differential ideal $I(f ; \Delta)$ generated by $f(x)$ in $F[x ; \Delta]$ coincides with the ordinary ideal in $F[X]$ generated by $f$ and all its derivatives $\partial_{1}^{i_{1}} \cdots \partial_{n}^{i_{n}}(f)$.

Note that if $f \notin F$ then $I(f ; \Delta)$ is proper: one may apply the notion of a characteristic set (see, e.g., [7, Chapter I.10]) or simply note that the set of all derivatives of $f$ is a Gröbner basis provided that we choose an ordering of monomials in such a way that highest derivative (leader) is contained in the leading monomial (e.g., rank ordering in [7, Chapter I.8]). Indeed, if uy is the leading monomial of $f(y \in X$ is the leader of $f, u$ is an ordered monomial in $X)$ then $u y^{\left(i_{1}, \ldots, i_{n}\right)}$ is the leading monomial of $\partial_{1}^{i_{1}} \cdots \partial_{n}^{i_{n}}(f)$. It is easy to see that there are no compositions (we follow the terminology of Shirshov [20], see [3] for details) among $f$ and its derivatives except for the case when $u y=y^{k}$, but in the latter case the only series of compositions of intersection of $f$ with itself is obviously trivial.

Hence, if $f \notin F$ then $I=I(f ; \Delta)$ is proper, and so is its radical $\sqrt{I}$. By the differential prime decomposition theorem (see, e.g., [15, Chapter 1]), $I=p_{1} \cap \cdots \cap p_{k}$, where $p_{i}$ are prime differential ideals in $F[x ; \Delta]$. In particular, $f \in p_{1}$, and $F[x ; \Delta] / p_{1}$ is a differential domain containing a root $x+p_{1}$ of $f$. Finally, the quotient field of that domain $Q\left(F[x ; \Delta] / p_{1}\right)$ is the desired differential field.

Therefore, every nontrivial equation over an arbitrary differential field $F$ has a solution in an extension $K$ of $F$. If $F$ is infinite then $K$ has the same cardinality as $F$, so the standard transfinite induction arguments similar to those applied to ordinary fields show that $F$ can be 
embedded into a differential field $\bar{F} \in \operatorname{Dif}_{n}$ in which every nontrivial differential polynomial has a root.

Corollary 4 ([13]). The Freiheitssatz holds for the variety of Poisson algebras.

Proof. Let $A_{2 n}=\mathbb{k}\left(x_{1}, y_{1}, x_{2}, y_{2}, \ldots, x_{n}, y_{n}\right)$ be the algebra of (commutative) rational functions over $\mathbb{k}, \partial_{i}=\partial_{x_{i}}, \partial_{i}^{\prime}=\partial_{y_{i}}$ be ordinary partial derivatives with respect to $x_{i}, y_{i}$, respectively. As $A_{2 n} \in \operatorname{Dif}_{2 n}$, there exists its algebraically closed extension $\bar{A}_{2 n} \in \operatorname{Dif}_{2 n}$. Let $\mathrm{PS}_{n}=A_{2 n}^{(\partial)}$ be the Poisson algebra defined by (1). Then $\mathrm{PS}_{n} \subseteq \bar{A}_{2 n}^{(\partial)}$, where the latter is a conditionally closed Poisson algebra by Proposition 1 .

It was shown in [5] that for every nonzero Poisson polynomial $h=h\left(x_{1}, \ldots, x_{m}\right), m \geq 1$, there exists a sufficiently large $N$ such that $\operatorname{PS}_{N}$ (and thus $\bar{A}_{2 N}^{(\partial)}$ ) does not satisfy the identity $h\left(x_{1}, \ldots, x_{m}\right)=0$. Lemma 1 implies the claim.

Let us twist the functor $\partial: \operatorname{Dif}_{2 n} \rightarrow$ Pois in order to obtain a conditionally closed generic Poisson algebra that does not satisfy a fixed polynomial identity.

Consider the variety $\mathrm{CDif}_{n}$ of commutative differential algebras with pairwise commuting derivations $\partial_{i}$ and constants $c_{i}, i=1, \ldots, n$, such that $\partial_{i}\left(c_{j}\right)=\delta_{i j}$. Then there exists a natural forgetful functor $\omega: \mathrm{CDif}_{n} \rightarrow \operatorname{Dif}_{n}$ erasing the information about constants.

In particular, $A_{2 n}$ may be considered as an algebra from $\operatorname{CDif}_{2 n}$ with derivations $\partial_{i}=\partial_{x_{i}}$, $\partial_{i}^{\prime}=\partial_{y_{i}}$, and constants $c_{i}=x_{i}, c_{i}^{\prime}=y_{i}, i=1, \ldots, n$. Moreover, if $A_{2 n} \subseteq A \in \operatorname{Dif}_{2 n}$ then $A=B^{(\omega)}$ for an appropriate $B \in \mathrm{CDif}_{2 n}$. Hence (see Remark 1), for every $n \geq 1$ there exists a conditionally closed algebra $\bar{B}_{2 n}$ in $\operatorname{CDif}_{2 n}, \bar{B}_{2 n}^{(\omega)}=\bar{A}_{2 n}$.

Suppose $B \in \mathrm{CDif}_{2 n}$ with derivations $\partial_{i}, \partial_{i}^{\prime}$ and constants $c_{i}, c_{i}^{\prime}, i=1, \ldots, n$. Let us consider the following functor $\tau$ from $\mathrm{CDif}_{2 n}$ to the variety $\mathrm{NDif}_{2 n}$ of commutative differential algebras with non-commuting derivations $\xi_{i}, \xi_{i}^{\prime}, i=1, \ldots, n$. On the same space $B$, define new derivations by

$$
\begin{aligned}
& \xi_{i}(a)=c_{i+1}^{\prime} \partial_{i}, \quad i=1, \ldots, n-1, \\
& \xi_{n}(a)=c_{1}^{\prime} \partial_{n}, \\
& \xi_{i}^{\prime}(a)=\partial_{i}^{\prime}(a), \quad i=1, \ldots, n,
\end{aligned}
$$

for $a \in B$. If $B$ is conditionally closed in $\operatorname{CDif}_{2 n}$ then $B^{(\tau)}$ is conditionally closed in NDif $2 n$.

Finally, define a functor $\xi$ from $\operatorname{NDif}_{2 n}$ to the variety GP of generic Poisson algebras by means of

$$
\{a, b\}=\sum_{i \geq 1} \xi_{i}(a) \xi_{i}^{\prime}(b)-\xi_{i}(b) \xi_{i}^{\prime}(a) .
$$

Denote by $\operatorname{GPS}_{n}$ the GP-algebra $\left(A_{2 n}^{(\tau)}\right)^{(\xi)}$.

Proposition 4. For every $n \geq 1$ there exists $N \geq 1$ such that the GP-algebra GPS $_{m}$ does not satisfy a polynomial identity of degree $n$ for all $m \geq N$.

Proof. Suppose $f \in \operatorname{GP}\left(t_{1}, t_{2}, \ldots\right)$ is a GP-polynomial of degree $n$ which is an identity on $\mathrm{GPS}_{m}$. By Proposition 2 there also exists a polylinear identity $g$ on $\mathrm{GPS}_{m}$ which is a Jacobian GP-polynomial.

Let us split $g$ into finely homogeneous components:

$$
g=g_{1}+\cdots+g_{k}
$$

each $g_{i}$ is a Jacobian GP-polynomial (but not an identity on $\mathrm{GPS}_{m}$ ). 
According to Corollary $3, g_{1}$ contains a summand $\alpha u_{1} \cdots u_{l}, \alpha \in \mathbb{k}^{*}$,

$$
u_{i}=\left\{t_{i_{1}}, \ldots\left\{t_{i_{m_{i}}}, t_{i_{m_{i}+1}}\right\} \ldots\right\}, \quad m_{i}=1,2 \text {. }
$$

Assume $m$ is large enough (e.g., $m>2 l$ ), and evaluate the variables in such a way that

$$
\begin{aligned}
& t_{i_{m_{i}+1}}=y_{k_{i}}, \quad t_{i_{m_{i}}}=x_{k_{i}}, \quad t_{i_{m_{i}-1}}=x_{k_{i}+1}, \quad \ldots, \quad t_{i_{1}}=x_{k_{i}+m_{i}-1}, \\
& k_{i+1} \geq k_{i}+m_{i}, \quad k_{l}+m_{l}<m .
\end{aligned}
$$

Then the only summand in $g_{1}\left(t_{1}, \ldots, t_{n}\right)$ is nonzero, namely, the summand mentioned in Corollary 3: it turns into $\alpha y_{k_{1}+m_{1}} \cdots y_{k_{l}+m_{l}} \neq 0$. Other $g_{i}^{\prime} s$ turn into zero.

Hence, $g$ cannot be a polynomial identity on $\mathrm{GPS}_{m}$.

Theorem 3. The Freiheitssatz holds for the variety of generic Poisson algebras.

Proof. Given $N \geq 1, G_{N}=\left(\bar{B}_{2 N}^{(\tau)}\right)^{(\xi)}$ is a conditionally closed algebra in GP by Proposition 1, and $\mathrm{GPS}_{N} \subseteq G_{N}$. The claim now follows from Proposition 4 and Lemma 1.

\section{Acknowledgements}

P. Kolesnikov was partially supported by FAPESP (grant 2012/04704-0) and RFBR (12-0133031), L. Makar-Limanov acknowledges the support of FAPESP (grant 2011/52030-5), and I. Shestakov was supported by FAPESP (grant 2010/50347-9) and CNPq (grant 3305344/20099). L. Makar-Limanov and I. Shestakov gratefully acknowledge Max-Planck-Institut für Mathematics, L. Makar-Limanov is also grateful to the University of Michigan and the Weizmann Institute of Science. The authors are grateful to Alexei Ovchinnikov for valuable discussions and to the referees for important remarks that helped to improve the exposition.

\section{References}

[1] Bokut L.A., Theorems of imbedding in the theory of algebras, Colloq. Math. 14 (1966), 349-353.

[2] Bokut L.A., Chen Y., Li Y., Lyndon-Shirshov basis and anti-commutative algebras, J. Algebra 378 (2013), 173-183, arXiv:1110.1264.

[3] Bokut L.A., Kolesnikov P.S., Gröbner-Shirshov bases: from inception to the present time, J. Math. Sci. 116 (2003), 2894-2916.

[4] Cherlin G., Model theoretic algebra - selected topics, Lecture Notes in Math., Vol. 521, Springer-Verlag, Berlin - New York, 1976.

[5] Farkas D.R., Poisson polynomial identities, Comm. Algebra 26 (1998), 401-416.

[6] Higman G., Scott E., Existentially closed groups, London Mathematical Society Monographs, New Series, Vol. 3, The Clarendon Press, Oxford University Press, New York, 1988.

[7] Kolchin E.R., Differential algebra and algebraic groups, Pure and Applied Mathematics, Vol. 54, Academic Press, New York - London, 1973.

[8] Kolesnikov P.S., Varieties of dialgebras, and conformal algebras, Sib. Math. J. 49 (2008), 257-272, math.QA/0611501.

[9] Kozybaev D., Makar-Limanov L., Umirbaev U., The Freiheitssatz and the automorphisms of free rightsymmetric algebras, Asian-Eur. J. Math. 1 (2008), 243-254, arXiv:0807.0608.

[10] Magnus W., Über discontinuierliche Gruppen mit einer definierenden Relation (Der Freiheitssatz), J. Reine Angew. Math. 163 (1930), 141-165.

[11] Makar-Limanov L., The skew field of fractions of the Weyl algebra contains a free noncommutative subalgebra, Comm. Algebra 11 (1983), 2003-2006.

[12] Makar-Limanov L., Algebraically closed skew fields, J. Algebra 93 (1985), 117-135. 
[13] Makar-Limanov L., Umirbaev U., The Freiheitssatz for Poisson algebras, J. Algebra 328 (2011), 495-503, arXiv:1004.2747.

[14] Mikhalev A.A., Shestakov I.P., PBW-pairs of varieties of linear algebras, Comm. Algebra 42 (2014), 667-687.

[15] Ritt J.F., Differential algebra, American Mathematical Society Colloquium Publications, Vol. 33, Amer. Math. Soc., New York, 1950.

[16] Romanovskii N.S., A theorem on freeness for groups with one defining relation in varieties of solvable and nilpotent groups of given degrees, Math. USSR Sb. 89 (1972), 93-99.

[17] Scott W.R., Algebraically closed groups, Proc. Amer. Math. Soc. 2 (1951), 118-121.

[18] Shestakov I.P., Quantization of Poisson superalgebras and the specialty of Jordan superalgebras of Poisson type, Algebra Logika 32 (1993), 309-317.

[19] Shestakov I.P., Speciality problem for Malcev algebras and Poisson Malcev algebras, in Nonassociative Algebra and its Applications (São Paulo, 1998), Lecture Notes in Pure and Appl. Math., Vol. 211, Dekker, New York, 2000, 365-371.

[20] Shirshov A.I., Some algorithmic problems for Lie algebras, Sib. Math. J. 3 (1962), 292-296.

[21] Shirshov A.I., Some algorithmic problems for $\varepsilon$-algebras, Sib. Math. J. 3 (1962), 132-137.

[22] Umirbaev U.U., On Schreier varieties of algebras, Algebra Logika 33 (1994), 180-193.

[23] Zhevlakov K.A., Slin'ko A.M., Shestakov I.P., Shirshov A.I., Rings that are nearly associative, Pure and Applied Mathematics, Vol. 104, Academic Press, Inc., New York - London, 1982. 